\title{
Влияние изотропного давления на вольт-амперную характеристику поверхностно-барьерных диодов $\mathrm{Sb}-p$-Si $\langle\mathrm{Mn}\rangle-\mathrm{Au}$
}

\author{
(C) С. Зайнабидинов, И.Г. Турсунов, О. Химматкулов
}

Национальный университет Узбекистана, 100174 Ташкент, Узбекистан

E-mail: ikromjon0804@gmail.com

(Получена 14 ноября 2017 г. Принята к печати 29 ноября 2017 г.)

Исследовано влияние гидростатического давления на вольт-амперные характеристики поверхностнобарьерных диодных структур типа $\mathrm{Sb}-p$ - $\mathrm{Si}\langle\mathrm{Mn}\rangle-\mathrm{Au}$. Определена высота потенциального барьера $\left(e \varphi_{\delta}=0.75\right.$ эВ) и барический коэффициент ее изменения $\left(\delta=-1.54 \cdot 10^{-11}\right.$ эВ/Па).

DOI: $10.21883 /$ FTP.2018.08.46215.8765

\section{1. Введение}

В последнее время все чаще применяются полупроводниковые тензодатчики с барьером Шоттки, отличающиеся высокой чувствительностью, широким частотным диапазоном, малыми размерами и простотой изготовления. Установлено, что поверхностно-барьерные диоды чувствительны к неоднородному давлению и в ряде случаев их чувствительность оказывается заметно выше, чем чувствительность $p-n$-переходов [1].

Исследование влияния давления на характеристики полупроводниковых приборов, в частности поверхностно-барьерных диодных структур, представляет интерес как для изучения тензоэффекта и механизмов его проявления в данных структурах с целью создания чувствительных к внешнему давлению приборов, так и для испытания надежности работы данных структур в условиях внешнего давления.

Цель настоящей работы - изучение вольт-амперной характеристики поверхностно-барьерных диодов $\mathrm{Sb}-p-\mathrm{Si}\langle\mathrm{Mn}\rangle-\mathrm{Au}$ при всестороннем гидростатическом давлении.

\section{2. Методика эксперимента}

Нами исследовано влияние всестороннего гидростатического сжатия (ВГС) в статическом режиме воздействия на вольт-амперную характеристику (BAX) поверхностно-барьерных диодов типа $\mathrm{Sb}-p$ - $\mathrm{Si}\langle\mathrm{Mn}\rangle-\mathrm{Au}$, в качестве базы которых использована компенсированная $\mathrm{Mn}$ кремниевая пластина дырочного типа проводимости с удельным сопротивлением $\rho=300$ Ом - см. Диодные структуры были изготовлены напылением золота и сурьмы на противоположные грани пластины с большой поверхностью, перпендикулярной к кристаллографической оси [111]. Выпрямляющий контакт создавался на границе раздела $\mathrm{Sb}-p$ - $\mathrm{Si}\langle\mathrm{Mn}\rangle$. Вольт-амперную характеристику поверхностно-барьерных диодов под давлением исследовали с помощью установки гидростатического давления с пневмоусилителем [2] в диапазоне давления $P=(0 \div 5) \cdot 10^{8}$ Па при температуре $T=300 \mathrm{~K}$.

\section{3. Результаты и их обсуждение}

На рис. 1 приведены ВАХ полученных диодных структур при значениях ВГС $P=0$ Па (кривая 1) и $P=5 \cdot 10^{8}$ Па (кривая 2) в статическом режиме воздействия. Видно, что ток как при прямом, так и при обратном напряжениях смещения растет пропорционально давлению. Токовая чувствительность к ВГС данных структур при одинаковых приложенных напряжениях в прямом направлении больше, чем в обратном.

Следует отметить, что чувствительность к ВГС по току, как при прямом, так и при обратном смещениях

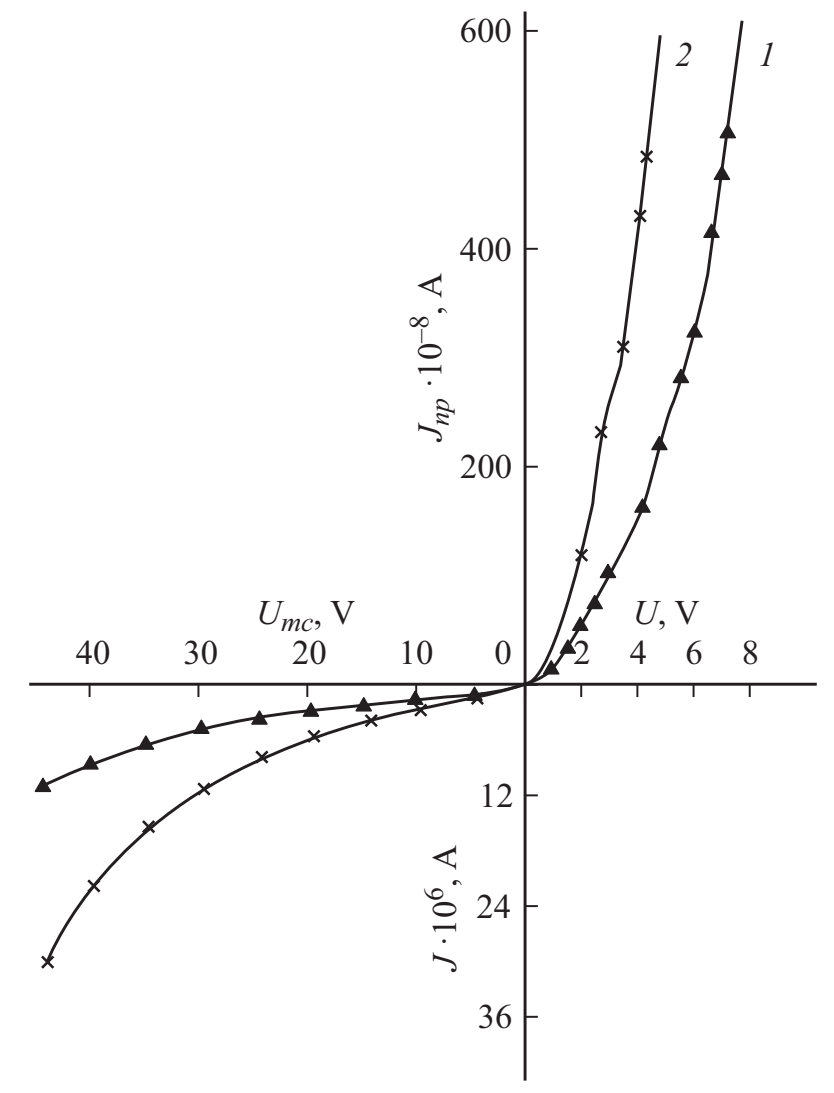

Рис. 1. ВАХ ПБД структур : $1-\mathrm{Sb}-p-\mathrm{Si}\langle\mathrm{Mn}\rangle-\mathrm{Au}$ : $1-P=0,2-P=5 \cdot 10^{8}$ Па. 


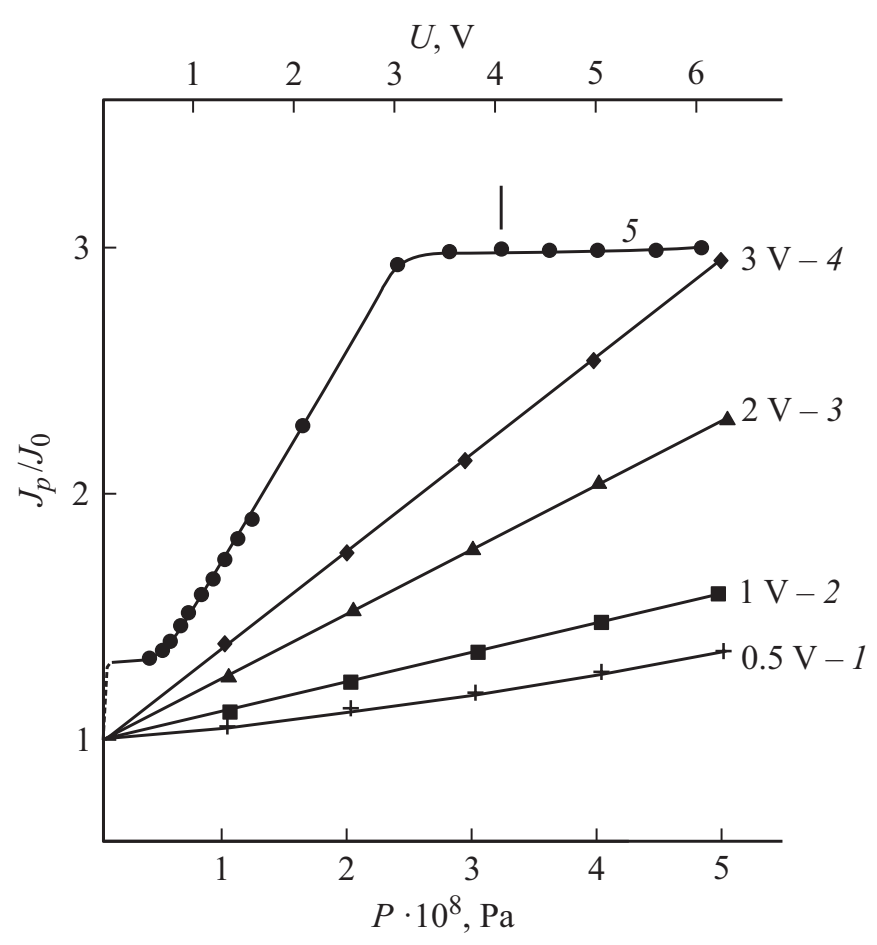

Рис. 2. Зависимость относительного изменения тока прямой ветви ВАХ ПБД $\mathrm{Sb}-p$ - $\mathrm{Si}\langle\mathrm{Mn}\rangle-\mathrm{Au}$ от статического ВГС (1-4) и напряжения (5).

зависит от значения приложенного напряжения. На рис. 2 приведены зависимости прямого тока структуры в относительных единицах от приложенного давления в диапазоне $P=(0 \div 5) \cdot 10^{6}$ Па при напряжениях смещения $U=0.5,1,2$ и $3 \mathrm{~B}$ и зависимость $J_{p} / J_{0}=f(U)$ при $P=5 \cdot 10^{6}$ Па. Видно, что тензотоки данных поверхностно-барьерных диодов (ПБД) сильно зависят от приложенного напряжения смещения $U$, при этом относительное значение токов $J_{p} / J_{0}$ возрастает с ростом $U$ (кривые $1-4)$. Из кривой 5 видно, что в зависимостях $J_{p} / J_{0}=f(U)$ наблюдаются три характерных участка. В диапазоне напряжений до $U=0.5 \mathrm{~B}$ токовая чувствительность структуры постоянна и равна $J_{p} / J_{0}=1.35$.

Далее с увеличением напряжения до $U=3$ В отношение $J_{p} / J_{0}$ линейно растет. С дальнейшим увеличением напряжения $(U \geq 3 \mathrm{~B})$ чувствительность по току данных структур не изменяется и $J_{p} / J_{0}=3$.

Как известно [3], перенос заряда через выпрямляющий контакт металл-полупроводник осуществляется в большинстве случаев основными носителями в отличие от $p-n$-переходов, где электрический ток обусловлен неосновными носителями. В ряде случаев, особенно для полупроводников $p$-типа, существенным оказывается и вклад инжекции носителей тока из металла в полупроводник, при этом значение коэффициента инжекции растет при увеличении напряжения $[4,5]$.

Плотность тока в обоих случаях через идеальный поверхностно-барьерный диод можно определить из вы- ражения [4]

$$
j=j_{0}[\exp (q U / k T-1)],
$$

где $j_{0}$ - плотность тока насыщения для термоэлектронной эмиссии:

$$
j_{0}=A^{*} T^{2} \exp \left(-q \varphi_{\delta} / k T\right) .
$$

где $\varphi_{\delta}$ - высота потенциального барьера.

Имеется ряд процессов, приводящих к отклонению BAX от идеальной (1). К ним относятся: рекомбинация и генерация носителей тока в области пространственного заряда (ОПЗ), снижение высоты потенциального барьера из-за электростатических сил изображения, туннелирование носителей тока через поверхностные состояния, наличие тонкой диэлектрической пленки между металлом и полупроводником [6]. Для всех этих процессов зависимость тока от напряжения при прямых смещениях может быть приближенно описана выражением

$$
J=J_{0}\left[\exp \left(\frac{q U}{n k T}\right)^{-1}\right],
$$

где $n$ - коэффициент неидеальности.

Механизм токопрохождения в исследованных диодах тоже зависит от приложенного напряжения. На рис. 3 приведены прямые ветви ВАХ поверхностно-барьерных диодов $\mathrm{Sb}-p$ - $\mathrm{Si}\langle\mathrm{Mn}\rangle-\mathrm{Au}$ в логарифмическом масштабе для двух значений давления: $P=0$ Па (кривая 1) и $P=5 \cdot 10^{8}$ Па (кривая 2). Видно, что прямая ВАХ данных ПБД аналогична ВАХ $p-n$-перехода, учитывающей сопротивление базы и уровень инжекции, состоит в основном из четырех участков. В области малых токов, когда основная часть приложенного напряжения падает на

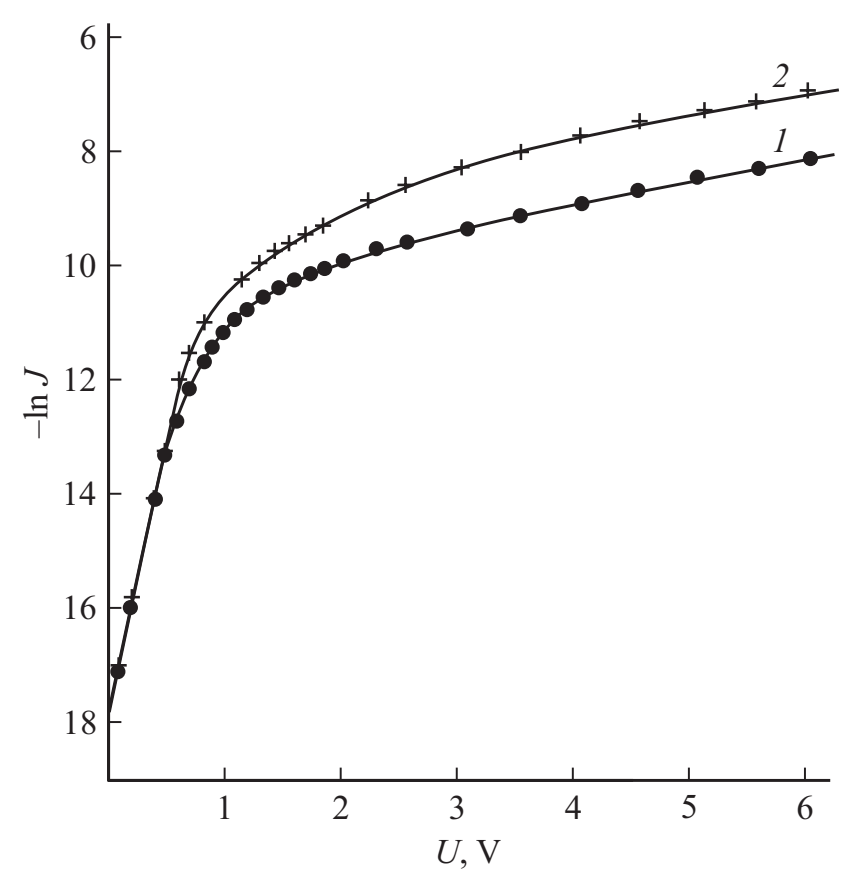

Рис. 3. Прямая ВАХ ПБД $\mathrm{Sb}-p-\mathrm{Si}\langle\mathrm{Mn}\rangle-\mathrm{Au}: 1-P=0$, $2-P=5 \cdot 10^{8}$ Па. 
сопротивлении барьера, т. е. в нашем случае $U<0.5$ В и $J_{p} / J_{0} \mathrm{BAX}$ можно описывать с помощью выражения (3). В координатах $\ln J$ от $U$ этот участок ВАХ представляет собой отрезок прямой линии с тангенсом угла наклона к оси напряжений, равным $q / n k T$ (рис. 3 ).

Далее с увеличением тока через диод падение напряжения на базе диода линейно растет, $U_{\sigma}=J R_{\sigma}$ и приложенное напряжение перераспределяется между базой и барьером Шоттки (БШ): $U=U_{0}+U_{\sigma m}$, где $U_{\sigma m}=k T \ln \left(J / J_{0}+1\right) / q$, так как база использованных ПБД является компенсированной. Этот участок ВАХ $(0.5<U<1 \mathrm{~B})$, где значение прямого тока под всесторонним давлением растет, описывается выражением

$$
J=J_{0}\left[\exp \left(q \frac{U-J R_{\sigma}}{k T}\right)^{-1}\right] .
$$

Затем должен быть участок, соответствующий переходу от низкого уровня инжекции к высокому $(1 \leq U \leq 3 \mathrm{~B})$.

Последний участок ВАХ поверхностно-барьерного диода $(U \geq 3 \mathrm{~B})$, где инжекция носителей тока играет существенную роль и токовая чувствительность имеет максимальное и постоянное значение, описывается, согласно [7], выражением

$$
J=J_{0} \exp \left(\frac{q U}{c k T}\right) .
$$

В координатах $\ln J$ от $U$ этот участок ВАХ представляет собой отрезок прямой линии с тангенсом угла наклона к оси $U$, равным $q / c k T$ (рис. 3 ).

В области малых токов, когда ВAX ПБД $\mathrm{Sb}-p-\mathrm{Si}\langle\mathrm{Mn}\rangle-\mathrm{Au}$ можно описывать выражением (3) согласно $[4,8]$, и можно вычислить высоту потенциального барьера с помощью выражения

$$
\varphi_{c}=\frac{k T}{q} \ln \frac{A^{*} T^{2}}{J_{0}},
$$

где $A^{*}=79.2$ - постоянная Ричардсона для дырок, $J_{0}$ - ток насыщения, определяемый из эксперимента. Расчеты показали, что постоянная Ричардсона при всестороннем сжатии до $P=5 \cdot 10^{8}$ Па изменяется всего на $\sim 1 \%$.

На рис. 4 приведена зависимость высоты потенциального барьера от значения приложенного ВГС в диапазоне давлений $P=(0 \div 5) \cdot 10^{8}$ Па и при $T=300 \mathrm{~K}$, вычисленная по формуле (6). При этом $J_{0}$ определялась, согласно [4], линейной экстраполяцией прямой ветви BAX $(U<0.5 \mathrm{~B})$ к $U=0 \mathrm{~B}$. Видно, что значение высоты потенциального барьера при $P=0$ Па $e \varphi_{\delta}=0.75 \mathrm{~B}$ и она линейно уменьшается с ростом ВГС. При этом барический коэффициент этого изменения составляет $\delta=-1.54 \cdot 10^{-11}$ эВ/Па и практически точно совпадает с литературными данными для барического коэффициента изменения ширины запрещенной зоны кремния, равной $-1.5 \cdot 10^{-11}$ эВ/Па [9]. Поэтому можно предположить, что изменение высоты барьера при ВГС в

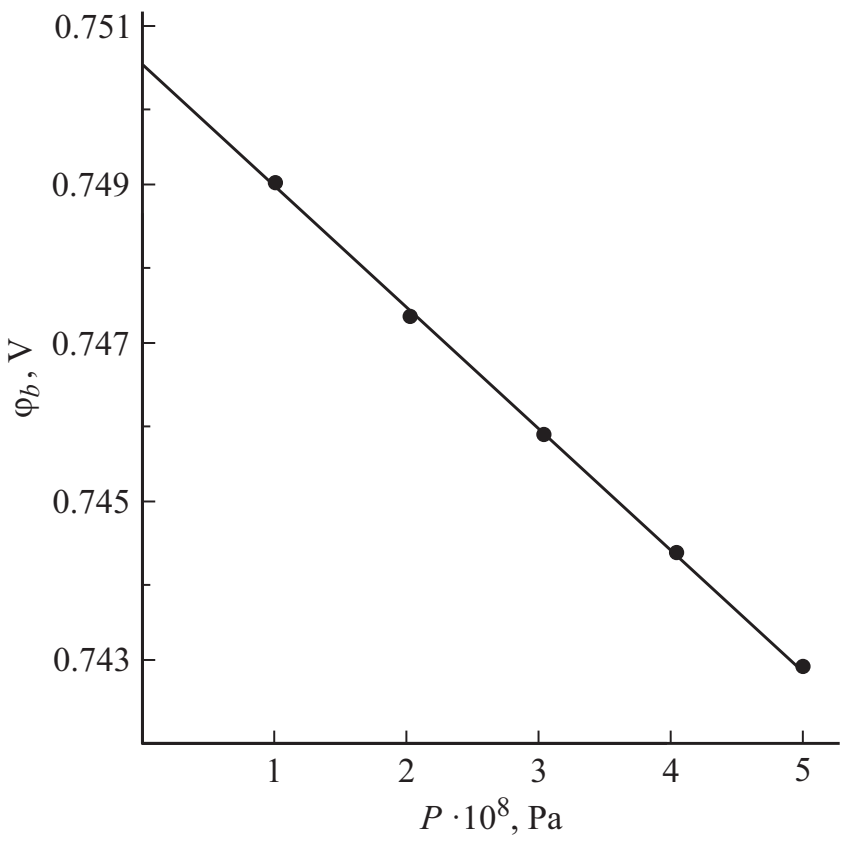

Рис. 4. Зависимость высоты потенциального барьера ПБД $\mathrm{Sb}-p-\mathrm{Si}\langle\mathrm{Mn}\rangle-\mathrm{Au}$ от статического ВГС.

данных структурах в основном обусловлено изменением ширины запрещенной зоны кремния.

Расчеты с использованием экспериментальных данных показали, что во всем диапазоне напряжений изменение прямого тока под ВГС нельзя объяснить только изменением концентрации дырок в базе диода.

Токовые чувствительности, определенные, согласно работе [9], как $\gamma=J_{0}^{-1} d J / d P$ и $\gamma=q(k T)^{-1} d \varphi_{\delta} / d P$, в области малых токов составили соответственно $6.56 \cdot 10^{-10}$ и $7.28 \cdot 10^{-10}$ Па, что позволяет предположить то, что при малых смещениях $J \leq 0.5$ В изменение тока обусловлено в основном изменением потенциального барьера.

Как было сказано выше, с увеличением тока через диод напряжение на базе диода растет и изменение проводимости базы под ВГС приводит к перераспределению приложенного в прямом направлении напряжения между базой и барьером структуры. Поэтому можно предположить, что к дальнейшему увеличению токовой чувствительности существенным является вклад положительной обратной связи в области БШ, т.е. под давлением происходит перераспределение напряжения и увеличение напряжения на барьере приводит к увеличению тока структуры $[10,11]$.

\section{4. Заключение}

На основе полученных результатов можно сделать следующие выводы:

1. Показано, что при $U_{d}<0.5 \mathrm{~B}$ основным механизмом переноса тока является термоэлектронная эмиссия 
носителей тока через барьер, и изменение прямого тока при ВГС обусловлено в основном изменением потенциального барьера. При напряжениях $0.5<U<3$ В изменение тока обусловлено в основном внутренней барической положительной обратной связью, проявляющейся при ВГС.

2. Определены высота потенциального барьера и барический коэффициент ее изменения, которые оказались равными $е \varphi_{\delta}=0.75$ эВ и $\delta=-1.54 \cdot 10^{-11}$ эВ/Па. Показано, что изменение высоты потенциального барьера при ВГС обусловлено изменением ширины запрещенной зоны $\mathrm{Si}$.

3. Следует отметить, что чувствительность как прямого, так и обратного токов к ВГС зависит от приложенного на диод напряжения, т.е. чувствительность растет с ростом приложенного напряжения. Чувствительность прямого тока к ВГС больше, чем чувствительность обратного тока при одинаковых напряжениях.

\section{Список литературы}

[1] М.И. Елинсон, В.И. Поколякин, А.Л. Полякова, Г.В. Степанов, В.В. Шкловская-Корди. РЭ, 15 (1), 210 (1970).

[2] А. Абдураимов, С.3. Зайнабидинов, О.О. Маматкаримов, О. Химматкулов, Т.Э. Худайбергенов. ПТЭ, 5, 229 (1988).

[3] В.И. Стриха, Е.В. Бузанева, И.А. Радзиевский. Полупроводниковые приборы с барьером Шоттки (М, Сов. радио, 1974).

[4] С. Зи. Физика полупроводниковых приборов (М, Мир, 1984), т. 1.

[5] Р. Смит. Полупроводники (М, Мир. 1982).

[6] А. Абдураимов, С. Зайнабидинов, О.О. Маматкаримов, А. Саидхонов, И.Г. Турсунов, О. Химматкулов. Узб. физ. журн., 5, 56 (1993).

[7] А. Абдураимов, С.3. Зайнабидинов, О.О. Маматкаримов, О. Химматкулов. Узб. физ. журн. 5, 47 (1993).

[8] А. Абдураимов, А. Тешабоев, О.О. Маматкаримов, О. Химматкулов. Тез. докл. I нац. конф. „Дефекты в полуполупроводниках“ (Санк-Петербург, 1992) с. 155.

[9] П.С. Киреев. Физика полупроводников (М., Высш. шк., 1969).

[10] И.М. Викулин, В.И. Стафеев. Физика полупроводниковых приборов (М., Радио и связь, 1990).

[11] А. Абдураимов, С.3. Зайнабидинов, О.О. Маматкаримов, О. Химматкулов. ФТП, 27 (7), 1216 (1993).

Редактор А.Н. Смирнов

\section{Ingluence of Isotropic Pressure on the volt-amper characntristics of surface barrier diodes $\mathbf{S b}-p-\mathbf{S i}\langle\mathbf{M n}\rangle-\mathbf{A u}$}

\author{
S. Zainabidinov, O. Khimmatkulov, I.G. Tursunov \\ National University of Uzbekistan, \\ 100174 Tashkent, Uzbekistan
}

Abstract The effect of hydrostatic pressure volt-ampere characteristics of the surfactant type diode structures $\mathrm{Sb}-p-\mathrm{Si}\langle\mathrm{Mn}\rangle-\mathrm{Au}$. The height of the potential barrier and the baric coefficient of its change were determined, which turned out to be equal to $e \varphi_{\delta}=0.75 \mathrm{eV}$ and $\delta=-1.54 \cdot 10^{-11} \mathrm{eV} / \mathrm{Pa}$. 